\title{
Dual-Band Adjustable and Reactive I/Q Generator With Constant Resistance for Down- and Up-Converters
}

\author{
Sheng-Che Tseng, Student Member, IEEE, Chinchun Meng, Member, IEEE, and Yueh-Ting Lee
}

\begin{abstract}
Adjustable and reactive in-phase/quadrature (I/Q) generators with constant resistance are proposed for the first time in this paper with the properties of low loss, dual-band implementation, and high quadrature accuracy. The quadrature phase property and input matching of the $I / Q$ generator can be achieved at all frequencies simultaneously by the constant-resistance $\mathrm{I} / \mathrm{Q}$ generator. However, the magnitude balance of the dual-band $I / Q$ generator is achieved at two designed frequencies. A 2.4/5.2-GHz $\mathrm{I} / \mathrm{Q}$ down-converter and a $2.4 / 5.7-\mathrm{GHz}$ single-sideband up-converter are fabricated using $0.35-\mu \mathrm{m} \mathrm{SiGe} \mathrm{BiCMOS} \mathrm{technology.}$ The dual-band I/Q generator along with two single-to-differential amplifiers is integrated to provide differential quadrature local oscillator signals for dual-band mixers. The magnitude imbalance and phase error between the $I$ and $Q$ channels of the down-converter are $<1 \%$ and $<1^{\circ}$, respectively, while the maximum sideband rejection ratio of the up-converter is up to $50 \mathrm{~dB}$. Additionally, the operation bandwidth (sideband rejection ratio > $30 \mathrm{~dB}$ ) is $200 \mathrm{MHz}$ at $2.4 \mathrm{GHz}$ and $720 \mathrm{MHz}$ at $5.7 \mathrm{GHz}$.
\end{abstract}

Index Terms-Down-converter, dual band, in-phase/quadrature (I/Q), SiGe BiCMOS, single sideband (SSB), SSB rejection ratio, up-converter.

\section{INTRODUCTION}

$\mathbf{T}$ HERE is a tendency towards multimode and multiband communication systems, such as the IEEE $802.11 \mathrm{a} / \mathrm{b} / \mathrm{g}$ wireless local area network (WLAN) combo system [1]. The communication ability and diversity enhance for users but the challenges also augment for designers. For the RF integrated circuits (RFICs), it is very difficult that the optimal performance of the matching, noise figure, or quadrature accuracy is carried out for multiple frequency bands. Much research has focused on dual-band low-noise amplifiers [2]. The dual-band input matching is achieved by using a high-order reactive matching network. However, generating dual-band quadrature signals from reactive elements is still a challenge.

Manuscript received August 29, 2007; revised March 12, 2008. First published June 24, 2008; last published August 8, 2008 (projected). This work was supported by the National Science Council of Taiwan, R.O.C., under Contract NSC 96-2752-E-009-001-PAE and Contract NSC 95-2221-E-009-043-MY3, by the Ministry of Economic Affairs of Taiwan under Contract 96-EC-17-A-05-S1020, and by the Ministry of Education (MoE) Aiming for the Top University and Elite Research Center Development (ATU) Plan under Contract 95W803.

The authors are with the Department of Communication Engineering, National Chiao Tung University, Hsinchu 300, Taiwan, R.O.C. (e-mail: ccmeng@mail.nctu.edu.tw).

Color versions of one or more of the figures in this paper are available online at http://ieeexplore.ieee.org.

Digital Object Identifier 10.1109/TMTT.2008.926526
The quadrature mechanism is widely used in the system architecture, for instance, sub-harmonic mixers [3], in-phase/quadrature (I/Q) down-converters, single-sideband (SSB) up-converters [4], and Weaver and Hartley transceivers [5]. There had been five types of quadrature generators. The first one is a polyphase filter, which is employed throughout the RFICs [6], [7]. Its phase accuracy improves with more $R C-C R$ sections. More than three sections are needed to resist the fabrication variation and to maintain precise quadrature signals. However, the resistive loss of the polyphase filter also increases. Besides, the polyphase filter is not suitable at high-frequency regimes because of its parasitics and the resistor self-cutoff frequency [6]. Using ring oscillators to generator quadrature signals is the second means [8], [9]. Although the ring oscillators have wide oscillation frequency in comparison with $L-C$ oscillators, their poor phase accuracy and phase noise deteriorate the performance of the entire transceiver [10]. Third, differential signals can be converted to quadrature signals by a divide-by-2 divider [11]. The input differential signals should operate with twice the desired frequency, and the oscillator with two times the desired frequency is not easily designed at high frequencies. The fourth way is to apply a quadrature coupler. The quadrature couplers, like a transmission line coupler and a Lange coupler, are often used in millimeter-wave applications [12]. However, the coupler size limits its usage in the RFICs. In spite of the size reduction of the spiral transmission line coupler, the phase accuracy and magnitude balance are not easily controlled and, hence, the performance is not good enough [13], [14].

The final solution is to directly construct a quadrature oscillator with a cross-coupling scheme between two differential oscillators. There are two types of coupling schemes-the fundamental coupling scheme and harmonic coupling scheme. The fundamental coupling scheme includes the top-series coupling scheme, bottom-series coupling scheme, and paralleled coupling scheme [15]. There is a tradeoff between phase noise and quadrature accuracy in this fundamental coupling scheme [16]. The good quadrature accuracy can be fulfilled at the cost of the phase-noise performance of the oscillators. In the harmonic coupling scheme, perfect quadrature signals can be obtained without phase noise degradation, but a differential amplifier or a transformer performing the coupling between oscillators should function at twice the oscillation frequency [17]. The quadrature ring oscillators, coupled oscillators, and dividers as quadrature generators are only employed at the local oscillator (LO) stage. 


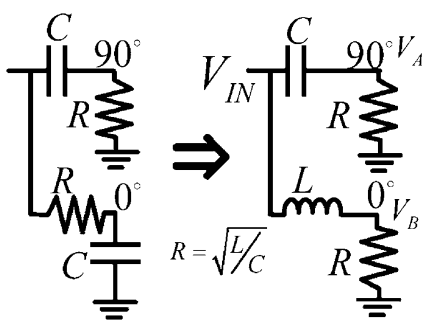

Fig. 1. Quadrature generators with the $C-R$ and $R-C$ sections or with the $C-R$ and $L-R$ sections.

The mentioned approaches are all utilized in a single frequency band. In this paper, a dual-band adjustable and constant-resistance I/Q generator is proposed. Since the generator is composed of pure reactive components, inductors, and capacitors, there is no loss introduced. Besides, the multiband I/Q generator is easily implemented by increasing the order of the $L-C$ networks. Capacitors along with varactors will add the ability to control the phase and magnitude of the quadrature outputs. The configuration and optimization for the quadrature accuracy are also described in this paper. A $2.4 / 5.2-\mathrm{GHz} \mathrm{I} / \mathrm{Q}$ down-conversion mixer and a 2.4/5.7-GHz SSB up-conversion mixer using $0.35-\mu \mathrm{m} \mathrm{SiGe} \mathrm{BiCMOS}$ are demonstrated with the dual-band adjustable and reactive I/Q generator. Thanks to the excellent quadrature accuracy, the magnitude mismatch and phase error of the down-converter outputs are $<1 \%$ and $<1^{\circ}$, respectively, and the maximum sideband rejection ratio of the up-converter is up to $50 \mathrm{~dB}$. According to the measurements, the down-converter outputs are always in quadrature and the I/Q generator input matching is achieved over all frequencies. In addition, the operation bandwidth (sideband rejection ratio $>30 \mathrm{~dB}$ ) is $200 \mathrm{MHz}$ at $2.4 \mathrm{GHz}$ and $720 \mathrm{MHz}$ at $5.7 \mathrm{GHz}$.

\section{REACTIVE I/Q GENERATOR}

A polyphase filter consisting of $R-C$ and $C-R$ sections is an I/Q generator. Nevertheless, the polyphase filter is not appropriate for high-frequency applications because the parasitics and the resistor self-cutoff frequency deteriorate phase and magnitude accuracy [6]. In this paper, the quadrature generator formed by $C-R$ and $L-R$ sections is proposed for the first time to the best of our knowledge. This reactive I/Q generator is derived from the conventional $C-R$ and $R-C$ sections, as shown in Fig. 1. For the $R-C$ section, the transfer function is derived as

where

$$
\frac{\frac{1}{S C}}{R+\frac{1}{S C}}=\frac{\frac{1}{R}}{\frac{1}{R}+S C}=\frac{R}{R+S C R^{2}}=\frac{R}{R+S L}
$$

$$
L=C R^{2} \text {. }
$$

Based on (1), the $R-C$ section can be replaced by the $L-R$ section, as shown in Fig. 1. Here, a reactive $\mathrm{I} / \mathrm{Q}$ generator is made of $C-R$ and $L-R$ sections and $R$ is given by the input impedance of the following stage. Thanks to no extra resistive components in this reactive quadrature generator, no resistive loss would be introduced.

\section{A. Reactive I/Q Generator With Constant Resistance}

The reactive I/Q generator is offered in this paper to replace the polyphase filter. The key point of design principle is that an inductor advances in phase, while a capacitor delays to result in $90^{\circ}$ phase difference. The reactive I/Q generator is shown in Fig. 1. $C-R$ and $L-R$ are in parallel. The voltage at nodes $A$ and $B$ in Fig. 1 can be expressed as

$$
V_{A}=\frac{R}{\left(\frac{1}{j \omega C}\right)+R} V_{\mathrm{IN}} \quad V_{B}=\frac{R}{j \omega L+R} V_{\mathrm{IN}} .
$$

From (2), the reactive I/Q circuit with $R=\omega_{0} L=\left(\omega_{0} C\right)^{-1}$ can generate truly balanced quadrature signals because $\mathrm{V}_{A}$ advances for $45^{\circ}$ and $\mathrm{V}_{B}$ delays $45^{\circ}$ with respect to $\mathrm{V}_{\mathrm{IN}} \cdot R=$ $\omega_{0} L=\left(\omega_{0} C\right)^{-1}$ can also be viewed as $R=(L / C)^{1 / 2}$ and $\omega_{0}=(L C)^{-1 / 2}$.

The input impedance of this generator can be written as

$$
\begin{aligned}
Z_{\text {in }} & =(R+j \omega L) / /\left(R+\frac{1}{j \omega C}\right) \\
& =\frac{R^{2}+\frac{L}{C}+R\left(j \omega L-\frac{j}{\omega C}\right)}{2 R+j \omega L-\frac{j}{\omega C}} \\
= & R
\end{aligned}
$$

when

$$
R=\sqrt{\frac{L}{C}}
$$

Under the condition of $R=(L / C)^{1 / 2}$, the input impedance is equal to $R$ regardless of frequencies. If the value of $R$ is equal to $50 \Omega$, the input port of the $\mathrm{I} / \mathrm{Q}$ generator is matched.

The voltage ratio between node $A$ and $B$ as a function of frequency can be expressed as

$$
\frac{V_{A}}{V_{B}}=\frac{j \omega L+R}{\left(\frac{1}{j \omega C}\right)+R}=\frac{\left(R^{2}-\frac{L}{C}\right)+j R\left(\omega L+\frac{1}{\omega C}\right)}{R^{2}+\left(\frac{1}{\omega C}\right)^{2}} .
$$

The voltage of node $A$ is always quadrature to that of node $B$ and unrelated to frequencies under the condition of $R=$ $(L / C)^{1 / 2}$. Also from (3), the input impedance is independent of frequency when $R=(L / C)^{1 / 2}$. However, the magnitude values of both nodes are identical only at frequency of $\omega_{0}$.

\section{B. Dual-Band and Adjustable I/Q Generator With Constant Resistance}

When compared with the polyphase filter, this reactive I/Q generator is easily designed for dual-band applications. A parallel $L-C$ and a series $L-C$ take place of $L$ and $C$ paths in the single-band I/Q generator, as shown in Fig. 2. The parallel $L-C$ section is inductive at low frequencies and capacitive at high frequencies. On the other hand, the series $L-C$ section has the opposite properties. Hence, this topology satisfies the quadrature condition at a low frequency $\left(\omega_{l}\right)$ and a high frequency $\left(\omega_{h}\right)$. The capacitor in Fig. 2 is in series with a varactor to add the tuning ability in the I/Q generator to conquer the fabrication 


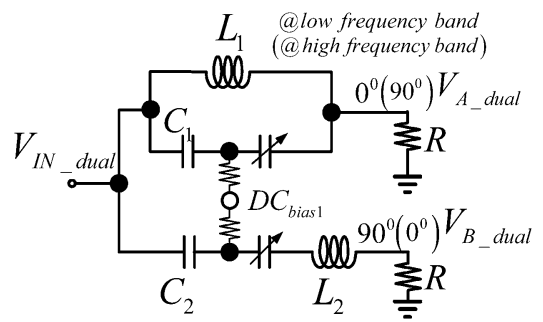

Fig. 2. Topology of the dual-band I/Q generator.

variation. For simplification in the analysis, the varactors are neglected. Based on the quadrature conditions,

$$
\begin{aligned}
& \left\{\begin{array}{l}
j \omega_{l} L_{1} / / \frac{1}{j \omega_{l} C_{1}}=j R \\
j \omega_{l} L_{2}+\frac{1}{j \omega_{l} C_{2}}=-j R
\end{array}\right. \\
& \left\{\begin{array}{l}
j \omega_{h} L_{1} / / \frac{1}{j \omega_{h} C_{1}}=-j R \\
j \omega_{h} L_{2}+\frac{1}{j \omega_{h} C_{2}}=j R
\end{array}\right.
\end{aligned}
$$

the inductance $\left(L_{1}\right.$ and $\left.L_{2}\right)$ and capacitance $\left(C_{1}\right.$ and $\left.C_{2}\right)$ are expressed in terms of $\omega_{l}, \omega_{h}$, and $R$ as follows:

$$
\begin{aligned}
L_{1} & =\frac{\omega_{h}-\omega_{l}}{\omega_{l} \omega_{h}} R \\
C_{1} & =\frac{1}{\left(\omega_{h}-\omega_{l}\right) R} \\
L_{2} & =\frac{1}{\omega_{h}-\omega_{l}} R
\end{aligned}
$$

and

$$
C_{2}=\frac{\omega_{h}-\omega_{l}}{\omega_{l} \omega_{h} R}
$$

TABLE I

CAlCulated INDUCTANCE and CAPACITANCE FOR DUAL-BAND WI-FI I/Q GENERATORS

\begin{tabular}{|c|c|c|c|c|}
\hline$\omega_{1}(\mathrm{GHz}) / \omega_{\mathrm{h}}(\mathrm{GHz}) / \mathrm{R}(\Omega)$ & $\mathrm{L}_{1}(\mathrm{nH})$ & $\mathrm{L}_{2}(\mathrm{nH})$ & $\mathrm{C}_{1}(\mathrm{pF})$ & $\mathrm{C}_{2}(\mathrm{pF})$ \\
\hline $2.4 / 5.2 / 50$ & 1.785 & 2.842 & 1.137 & 0.714 \\
\hline $2.4 / 5.7 / 50$ & 1.92 & 2.411 & 0.965 & 0.768 \\
\hline
\end{tabular}

The input impedance of the dual-band I/Q generator is derived as (10), shown at the bottom of this page.

Under the condition of $R=\left(L_{1} / C_{2}\right)^{1 / 2}=\left(L_{2} / C_{1}\right)^{1 / 2}$, the input impedance is also equal to $R$ regardless of frequencies, and the wideband matching can be achieved [18]. The voltage ratio at node $A_{-}$dual and $B \_d u a l$ in Fig. 2 can be expressed as (11), shown at the bottom of this page, and two nodes are always in quadrature regardless of frequencies when $R=$ $\left(L_{1} / C_{2}\right)^{1 / 2}=\left(L_{2} / C_{1}\right)^{1 / 2}$.

In Table $\mathrm{I}$, the ideal inductance and capacitance of the dual-band I/Q generator are calculated for WLAN applications. Here, the quadrature condition is designed based on the matching condition $(R=50 \Omega)$. The inductance and capacitance are reasonable and the dual-band I/Q generator can be realized in WLAN systems. Fig. 3 represents the magnitude ratio $\left(\left|V_{A_{-} \text {dual }} / V_{B_{-} \text {dual }}\right|\right)$ and phase error of the dual-band $\mathrm{I} / \mathrm{Q}$ generator with respect to normalized frequency $\omega_{0}=\omega_{l}$, when $R=\left(L_{1} / C_{2}\right)^{1 / 2}=\left(L_{1} / C_{2}\right)^{1 / 2}$. For the $2.4 / 5.2-\mathrm{GHz}$ application, $\omega_{h}=2.167 \omega_{l}$ and the magnitude error is smaller than $1 \mathrm{~dB}$ within the $0.96-1.04$ and 2.08-2.27 normalized frequency. For the 2.4/5.7-GHz application, $\omega_{h}=2.375 \omega_{l}$ and the magnitude error is smaller than $1 \mathrm{~dB}$ within the $0.96-1.04$ and 2.27-2.5 normalized frequency. At the transition frequency,

$$
\omega_{\text {transition }}=\sqrt{\omega_{l} \omega_{h}}=\frac{1}{\sqrt{L_{1} C_{1}}}=\frac{1}{\sqrt{L_{2} C_{2}}}
$$

$$
\begin{aligned}
Z_{\text {in_dual }} & =\left(R+j \omega L_{1} / / \frac{1}{j \omega C_{1}}\right) / /\left(R+j \omega L_{2}+\frac{1}{j \omega C_{2}}\right) \\
& =\frac{R^{2}+\left(\frac{L_{1}}{C_{2}} \frac{1-\omega^{2} L_{2} C_{2}}{1-\omega^{2} L_{1} C_{1}}\right)+j R\left(\frac{\omega L_{1}}{1-\omega^{2} L_{1} C_{1}}-\frac{1-\omega^{2} L_{2} C_{2}}{\omega C_{2}}\right)}{2 R+j\left(\frac{\omega L_{1}}{1-\omega^{2} L_{1} C_{1}}-\frac{1-\omega^{2} L_{2} C_{2}}{\omega C_{2}}\right)} \\
& =R
\end{aligned}
$$

when

$$
R=\sqrt{\frac{L_{1}}{C_{2}}}=\sqrt{\frac{L_{2}}{C_{1}}}
$$

$$
\frac{V_{A \_ \text {dual }}}{V_{B \_ \text {dual }}}=\frac{R-j \frac{1-\omega^{2} L_{2} C_{2}}{\omega C_{2}}}{R+j \frac{\omega L_{1}}{1-\omega^{2} L_{1} C_{1}}}=\frac{\left(R^{2}-\frac{L_{1}}{C_{2}} \frac{1-\omega^{2} L_{2} C_{2}}{1-\omega^{2} L_{1} C_{1}}\right)-j R\left(\frac{\omega L_{1}}{1-\omega^{2} L_{1} C_{1}}+\frac{1-\omega^{2} L_{2} C_{2}}{\omega C_{2}}\right)}{R^{2}+\left(\frac{\omega L_{1}}{1-\omega^{2} L_{1} C_{1}}\right)^{2}}
$$




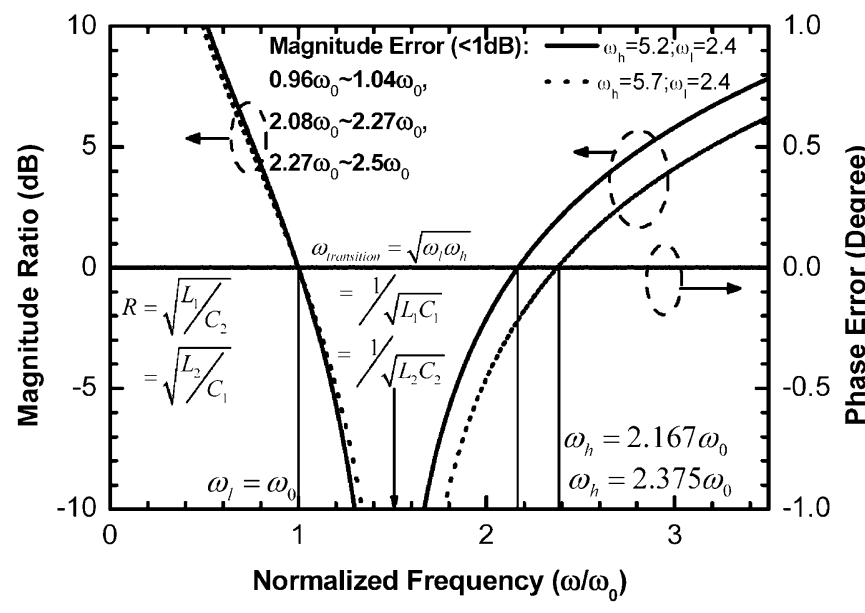

Fig. 3. Magnitude ratio $\left(\left|V_{A_{-} \text {dual }} / V_{B_{-} \text {dual }}\right|\right)$ and phase error of the dual-band reactive I/Q generator with respect to normalized frequency $\omega_{0}=\omega_{l}$.

the parallel $L-C$ network $\left(L_{1}\right.$ and $C_{1}$ ) is open, while the series $L-C$ network ( $L_{2}$ and $C_{2}$ ) is short. All of the power flows to node $B$ _dual. Therefore, the output magnitude ratio becomes zero and the phase reversal occurs.

In comparison with a polyphase filter, there are several advantages in this reactive I/Q generator, which are listed below.

1) The generator has low loss because of the absence of resistors and is suitable for high-frequency applications.

2) $\operatorname{Zin}=R$, and the input matching condition is determined by the input impedance of the following circuits. Hence, it is possible to achieve the wideband matching.

3) By increasing the order of the $L-C$ networks, it is easy to construct a multiband quadrature generator.

\section{Circuit Design}

The proposed dual-band adjustable I/Q generator is employed at the LO stage in an I/Q down-converter and an SSB up-converter. The entire schematics of the I/Q down-converter and the SSB up-converter are depicted in Figs. 4 and 5, respectively. The mixer topology is a micromixer, which has the wideband input matching property [19]-[21]. Thus, the micromixer is suitable for multiband or wideband applications. The quadrature differential LO signals are generated by the dual-band reactive I/Q generator and two single-to-differential amplifiers. Here, the single-to-differential input stage of the micromixer is adopted in the single-to-differential amplifier. Thus, the input impedance of the single-to-differential amplifier also serves as the loading impedance of the I/Q generator $R$. The emitter followers are employed as output buffers to drive $50-\Omega$ measurement equipments. The circuit topologies of the down- and up-converters are very similar, except the connection in the mixer outputs.

The phase property of LO signals is changed between dual bands, which alternates the quadrature property of the down-converter and the sideband property of the up-converter, as shown in Figs. 4 and 5. In the up-converter, the RF output signal of the low-frequency band is located at the upper sideband, while that of the high-frequency band is located at the lower sideband.

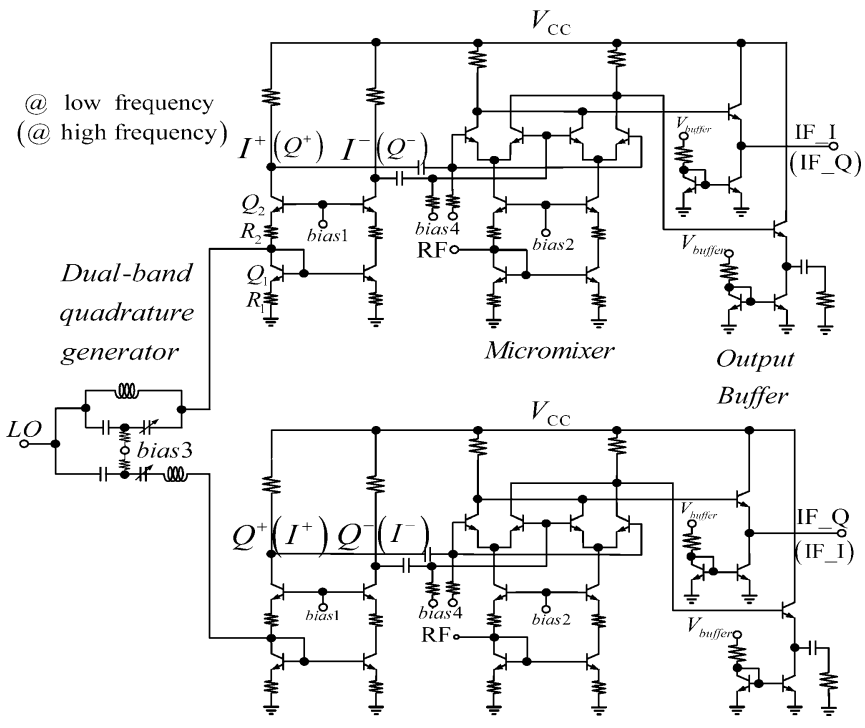

Fig. 4. Schematic of the dual-band I/Q down-converter.

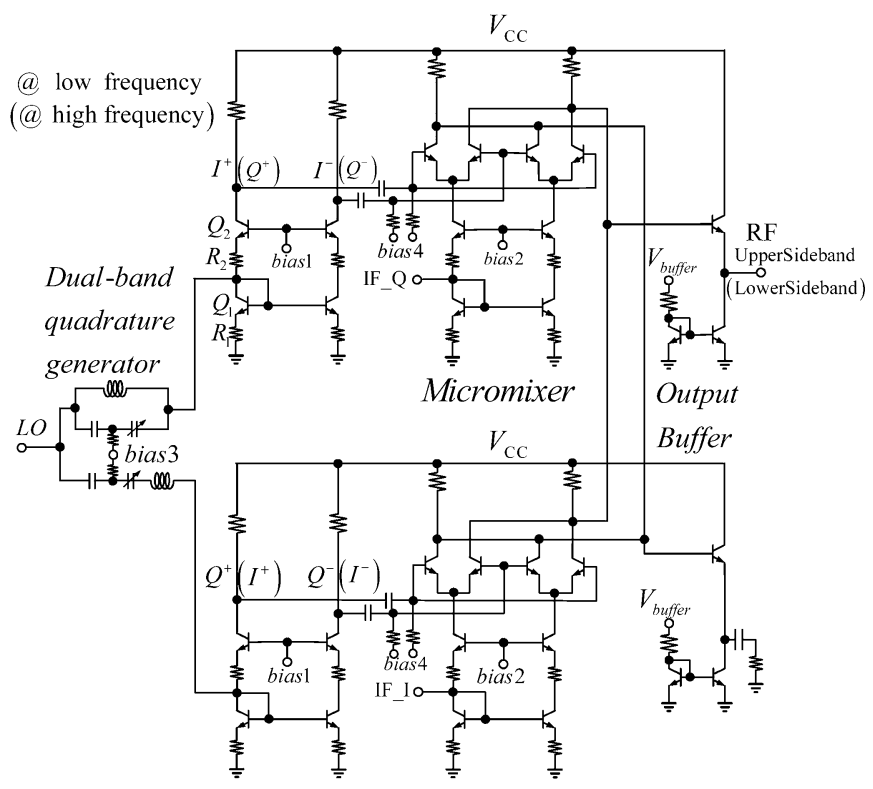

Fig. 5. Schematic of the dual-band SSB up-converter.

The adjustable mechanism is added to overcome the inductance and capacitance variations in integrated circuit (IC) fabrication. Fig. 6 illustrates four I/Q mismatch conditions. $A$ and $B$ denote the impedance of inductive $(R+j \omega L)$ and capacitive $(R+1 / j \omega C)$ sections, respectively. Obviously, the magnitude difference can be controlled by varactors as the quadrature phase is determined by the input impedance of the amplifier $R$, where the input impedance of the single-to-differential converter is approximately $\left(1 / \mathrm{gm}_{1}+R_{1}\right) / /\left(1 / \mathrm{gm}_{2}+R_{2}\right)$. Thus, the bias of the amplifier can control current and then the input impedance. Table II summarizes the configurations for four I/Q mismatch conditions, where $Z_{\mathrm{CAP}}$ is the impedance of the varactor.

\section{MEASUREMEnt Results}

A dual-band I/Q down-converter and a dual-band SSB up-converter are fabricated using the $0.35-\mu \mathrm{m}$ SiGe BiCMOS process. Both mixers utilize the dual-band adjustable and 


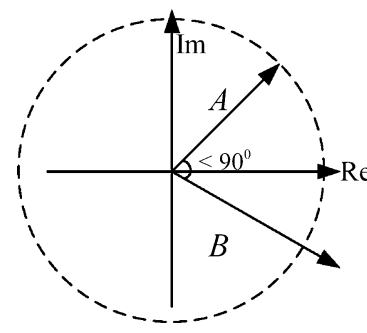

(a)

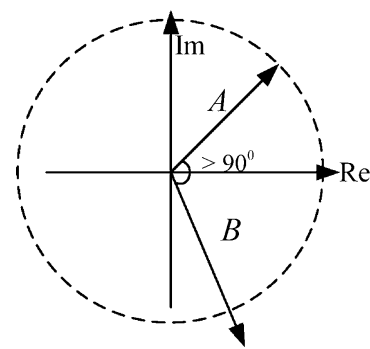

(c)

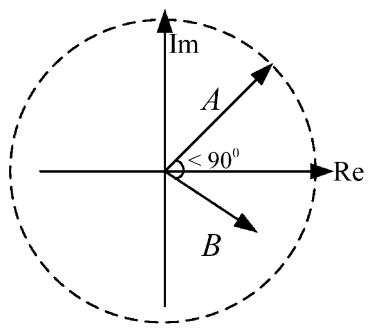

(b)

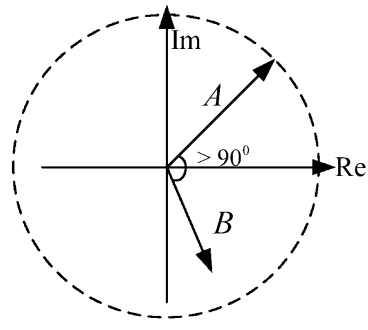

(d)
Fig. 6. Four types of $\mathrm{I} / \mathrm{Q}$ mismatch conditions. (a) $|A|<|B|$ and $\angle A B<90^{\circ}$. (b) $|A|>|B|$ and $\angle A B<90^{\circ}$. (c) $|A|<|B|$ and $\left.\angle A B\right\rangle$ $90^{\circ}$. (d) $|A|>|B|$ and $\angle A B>90^{\circ}$.

TABLE II

AdJustable Mechanism for Four I/Q Mismatch Conditions

\begin{tabular}{|c|c|c|}
\hline $\mathrm{I} / \mathrm{Q}$ mismatch conditions & $R$ & $Z_{C A P}$ \\
\hline$|A|<|\mathrm{B}| \& \angle \mathrm{AB}<90^{\circ}$ & $\searrow$ & $\searrow$ \\
\hline$|A|>|\mathrm{B}| \& \angle \mathrm{AB}<90^{\circ}$ & $\searrow$ & $\nearrow$ \\
\hline$|A|<|\mathrm{B}| \& \angle \mathrm{AB}>90^{\circ}$ & $\nearrow$ & $\searrow$ \\
\hline$|A|>|\mathrm{B}| \& \angle \mathrm{AB}>90^{\circ}$ & $\nearrow$ & $\nearrow$ \\
\hline
\end{tabular}

reactive I/Q generators to provide accurate quadrature $\mathrm{LO}$ signals. The die photographs are displayed in Figs. 7 and 8, respectively. The dual-band LO I/Q generator occupies the estate of $600 \mu \mathrm{m} \times 300 \mu \mathrm{m}$.

\section{A. 2.4- and 5.2-GHz I/Q Down-Converter}

This down-conversion mixer operates at $2.4 / 5.2 \mathrm{GHz}$ with the voltage supply of $3.3 \mathrm{~V}$. The capacitances of the I/Q generator is varied by tuning the varactor capacitance and, hence, the optimal performance is achieved. The power consumption of the down-converter at 2.4 and $5.2 \mathrm{GHz}$ is 37.9 and $33.3 \mathrm{~mW}$, respectively.

The conversion gain of the I and Q channels is dependent on the LO frequency, as shown in Fig. 9. The matched point between the I and Q channels occurs at 2.4 and $5.2 \mathrm{GHz}$. The conversion gain at $2.4 / 5.2 \mathrm{GHz}$ is approximately $5.2 / 1.2 \mathrm{~dB}$. At frequency below the matched point frequency, the inductive path of the I/Q generator obtains more power than the capacitive path does. Therefore, the I-channel conversion gain is higher. On the contrary, at frequency above the matched point frequency, the Q-channel down-converter has a higher conversion gain.

At $2.4 \mathrm{GHz}$, the mixer is driven with $\mathrm{LO}$ power of $-3 \mathrm{dBm}$ and has $-10.5-\mathrm{dBm} \mathrm{IP}_{1 \mathrm{~dB}},-2-\mathrm{dBm} \mathrm{IIP}_{3}, 900-\mathrm{MHz} 3-\mathrm{dB}$ IF bandwidth, -21.65-dB RF input return loss, $-12.45-\mathrm{dB}$ LO input return loss, -9.71-dB IF input return loss, and 19-dB noise

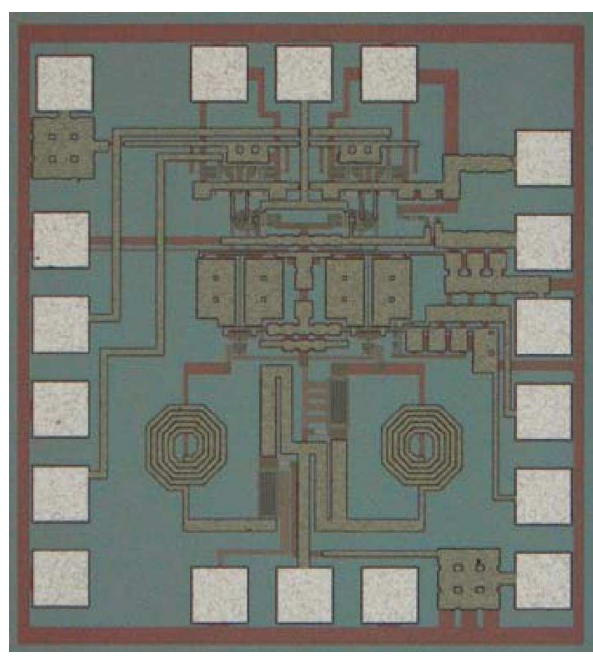

Fig. 7. Die photograph of the dual-band I/Q down-conversion mixer.

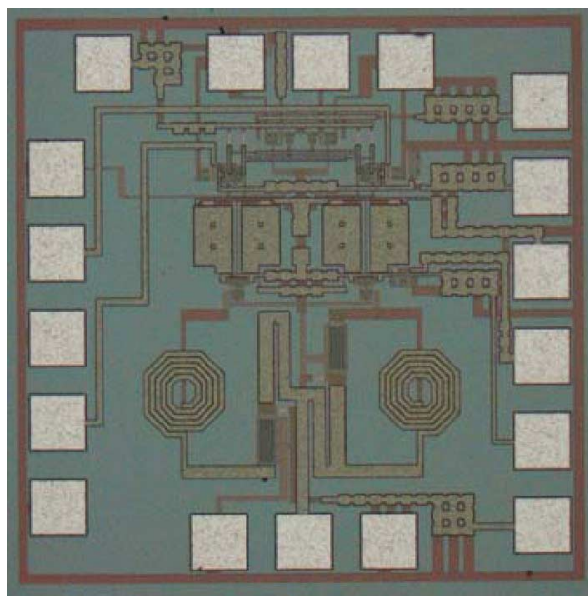

Fig. 8. Die photograph of the dual-band SSB up-conversion mixer.

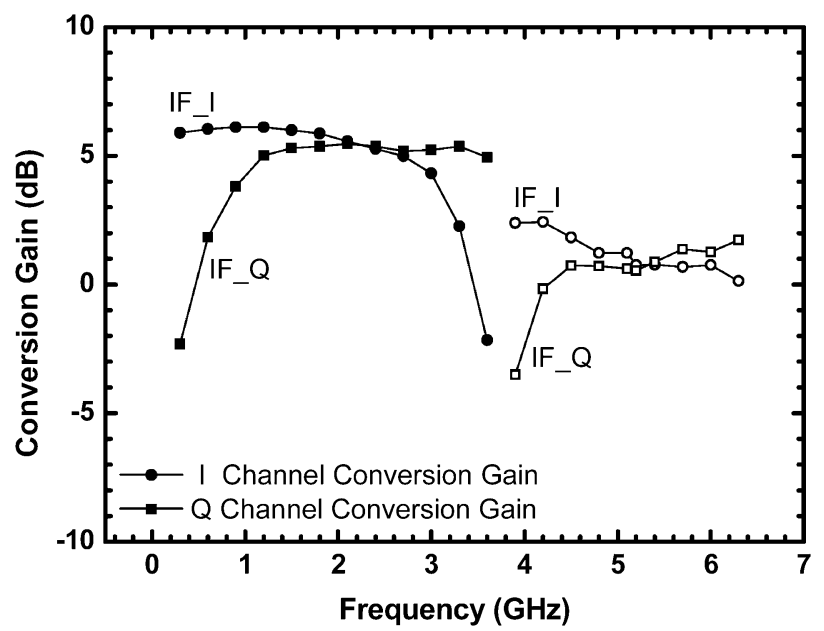

Fig. 9. Conversion gain of I and Q channels of the dual-band I/Q down-conversion mixer.

figure. The LO input port is single-ended. The reactive I/Q generators is lossless and does not increase the $\mathrm{LO}$ driven power. The rms magnitude error is approximately $0.204 \%$ and phase error is approximately $0.2^{\circ}$, which shows excellent orthogonal property of two outputs. 


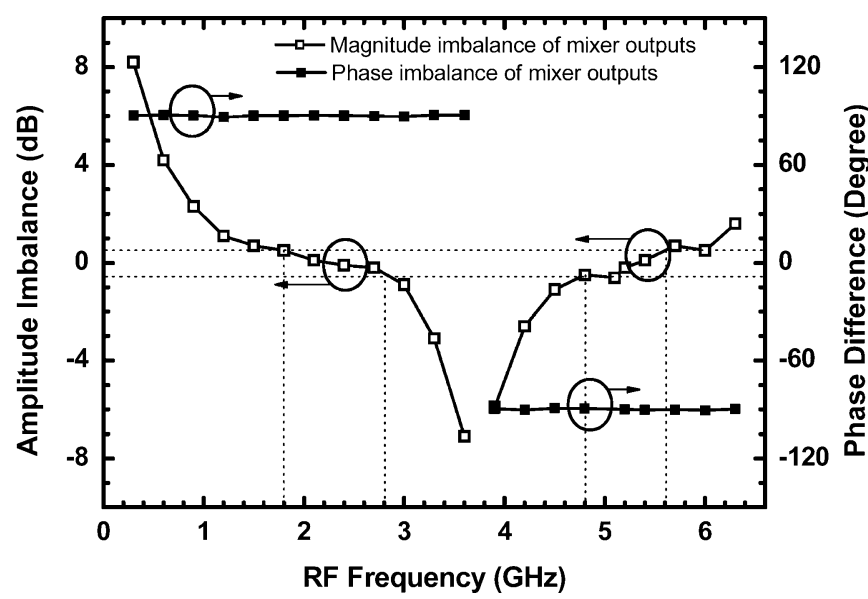

Fig. 10. Output phase and amplitude balance for the dual-band I/Q downconverter.

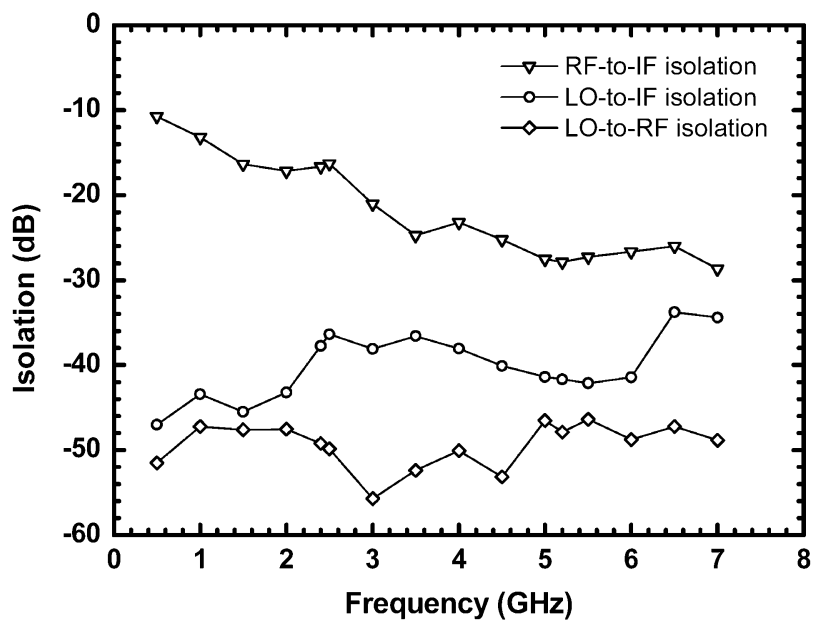

Fig. 11. Port-to-port isolations of the dual-band I/Q down-converter.

At $5.2 \mathrm{GHz}$, the RF, LO, and IF return loss of the mixer are $-17.3,-11.78$, and $-10.28 \mathrm{~dB}$, respectively. The $\mathrm{IP}_{1 \mathrm{~dB}}$ and $\mathrm{IIP}_{3}$ are -9.5 and $-0.5 \mathrm{dBm}$, respectively. The LO power is $0.1 \mathrm{dBm}$. The $3-\mathrm{dB}$ IF bandwidth is the same as that at $2.4 \mathrm{GHz}$. The noise figure is approximately $21 \mathrm{~dB}$. This I/Q mixer has $0.944 \%$ gain mismatch and $0.68^{\circ}$ phase error at $5.2-\mathrm{GHz} R F$ frequency. Fig. 10 describes the output phase and amplitude balance with respect to RF frequency. The phase error is smaller than $1^{\circ}$ over the entire frequency range and is limited by the measurement accuracy of $\pm 0.5^{\circ}$ phase and $\pm 0.1-\mathrm{dB}$ amplitude error. The measurement results agree with the discussion in Section II. The phase difference is always in quadrature and the phase reversal occurs at the transition frequency of around 3.6 GHz. Within $\pm 0.5-\mathrm{dB}$ output magnitude imbalance and $1^{\circ}$ phase error, this down-converter works from 1.8 to $2.8 \mathrm{GHz}$ and from 4.8 to $5.6 \mathrm{GHz}$ for each band. The operation bandwidth of the mixer is larger than that of the reactive I/Q generator because the conversion gain keeps constant and is insensitive to the LO power as long as the LO power is large enough to switch the Gilbert cell [20]. As shown in Fig. 11, the LO-to-IF, RF-to-IF, and LO-to-RF isolations are below $-35,-15$, and $-45 \mathrm{~dB}$, respectively.

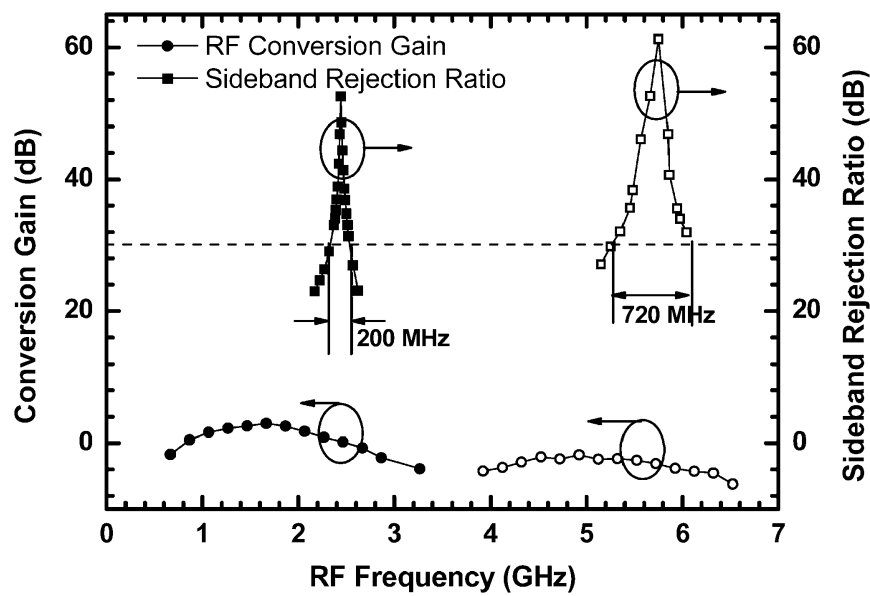

Fig. 12. Conversion gain and sideband rejection ratio of the up-converter.

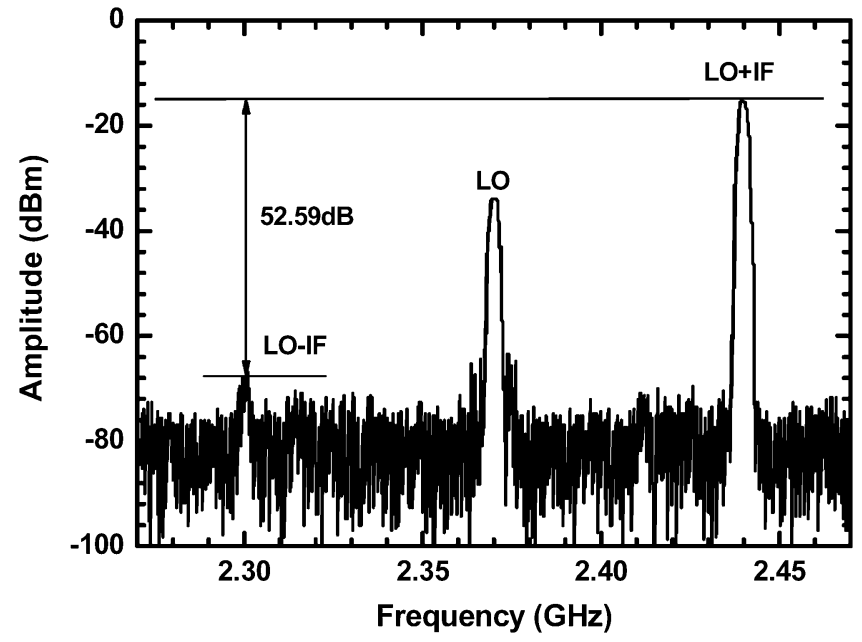

Fig. 13. Output spectrum of the dual-band SSB up-converter at $2.4 \mathrm{GHz}$. The sideband rejection ratio is $52.59 \mathrm{~dB}$.

\section{B. 2.4- and 5.7-GHz SSB Up-Converter}

The dual-band adjustable and reactive I/Q generator is also employed in the 2.4/5.7-GHz SSB up-converter. As shown in Fig. 12, the conversion gain at 2.4 and $5.7 \mathrm{GHz}$ are 0.5 and $-2.2 \mathrm{~dB}$, respectively. The peaks of the SSB rejection ratio occur at 2.4 and $5.7 \mathrm{GHz}$. The peak values at 2.4 and $5.7 \mathrm{GHz}$ are 52.59 and $62.85 \mathrm{~dB}$, respectively. The sideband rejection bandwidth ( $>30-\mathrm{dB}$ rejection ratio) is approximately $200 \mathrm{MHz}$ at $2.4 \mathrm{GHz}$ and $700 \mathrm{MHz}$ at $5.7 \mathrm{GHz}$. The $30-\mathrm{dB}$ sideband rejection ratio represents approximately $0.6-\mathrm{dB}$ magnitude error with perfect quadrature phase [22]. The output signal is located at the upper sideband at the $2.4 \mathrm{GHz}$ and located at the lower sideband at $5.7 \mathrm{GHz}$, as shown in Figs. 13 and 14 because the phase property of the I/Q generator between $2.4-5.7 \mathrm{GHz}$ is exchanged. The transition frequency is approximately $3.7 \mathrm{GHz}$. The performance of the sideband rejection is very sensitive to the phase and amplitude balance of the I/Q generator. The high sideband rejection ratio performance reveals that this adjustable and reactive I/Q generator can provide excellent quadrature outputs with equal power for dual-band applications.

At 2.4-GHz RF frequency, with the LO power of $-2.5 \mathrm{dBm}$, the mixer has $\mathrm{OP}_{1 \mathrm{~dB}}$ of $-7 \mathrm{dBm}, \mathrm{OIP}_{3}$ of $4 \mathrm{dBm}$, and the total 


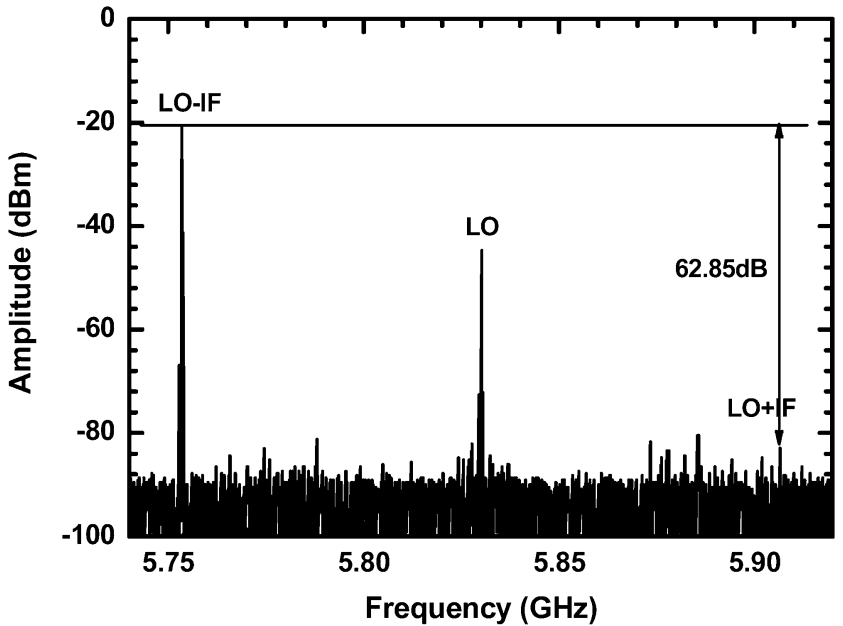

Fig. 14. Output spectrum of the dual-band SSB up-converter at $5.7 \mathrm{GHz}$. The sideband rejection ratio is $62.85 \mathrm{~dB}$.

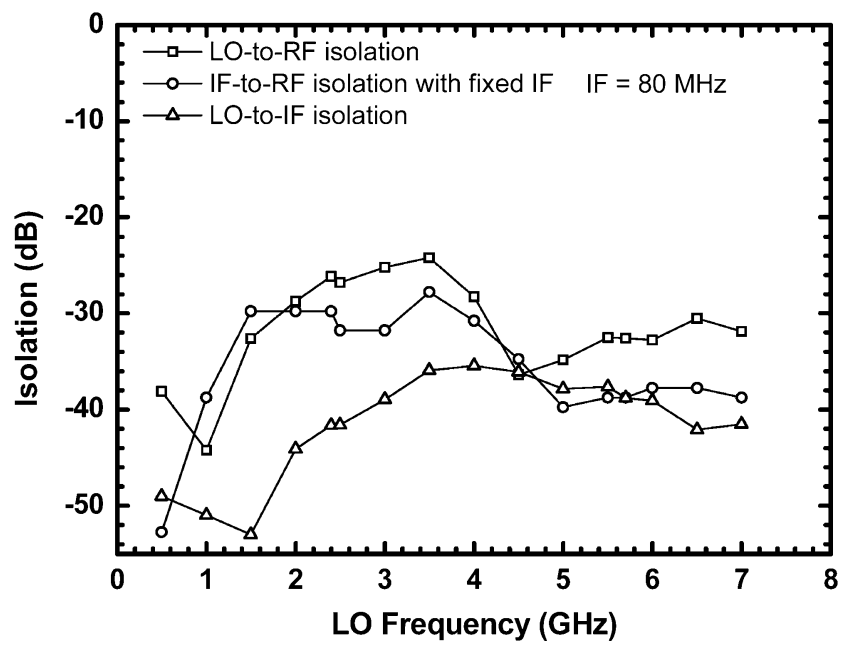

Fig. 15. Port-to-port isolations of the dual-band SSB up-converter.

power consumption of $38 \mathrm{~mW}$. At $5.7 \mathrm{GHz}$, with the $\mathrm{LO}$ power of $7.5 \mathrm{dBm}$, the mixer has $\mathrm{OP}_{1} \mathrm{~dB}$ of $-6.5 \mathrm{dBm}$ and $\mathrm{OIP}_{3}$ of $4 \mathrm{dBm}$. Fig. 15 illustrates the LO-to-RF and IF-to-RF isolations. The LO-to-RF isolation is below $-24 \mathrm{~dB}$ and the IF-to-RF isolation measured with the fixed IF of $80 \mathrm{MHz}$ is below $-28 \mathrm{~dB}$. In addition, the LO-to-IF isolation is approximately -41 and $-38 \mathrm{~dB}$ for 2.4- and 5.7-GHz bands, respectively.

The LO return loss of the dual-band I/Q down-converter and SSB up-converter is displayed in Fig. 16. Obviously, the wideband matching is achieved as expected because of the constant resistance property of the dual-band I/Q generator.

Table III summarizes the measured performance of both mixers. According to the measured results, this dual-band adjustable and reactive I/Q generator has optimal quadrature performance to achieve excellent quadrature outputs for a down-conversion mixer and high sideband rejection ratio for an up-converter. Compared with a polyphase filter, this reactive $\mathrm{I} / \mathrm{Q}$ generator has no resistive loss and achieves dual-band operations easily.

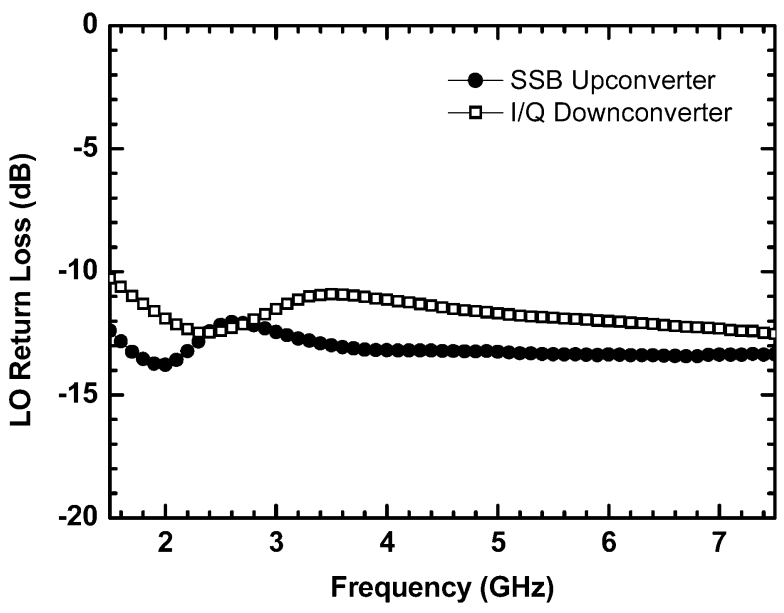

Fig. 16. LO return loss of the dual-band I/Q down-converter and SSB upconverter.

TABLE III

TUNABLE DUAL-BAND I/Q DOWN-CONVERTER AND SSB UP-CONVERTER

\begin{tabular}{|c|c|c|c|c|}
\hline \multicolumn{5}{|c|}{ TSMC 0.35um 3P3M SiGe BiCMOS } \\
\hline Item & \multicolumn{2}{|c|}{$\overline{\mathrm{UP}}$} & \multicolumn{2}{|c|}{ DOWN } \\
\hline Frequency & $2.4 \mathrm{GHz}$ & $5.7 \mathrm{GHz}$ & $2.4 \mathrm{GHz}$ & $5.2 \mathrm{GHz}$ \\
\hline $\begin{array}{c}\text { Conversion } \\
\text { Gain }\end{array}$ & $0.5 \mathrm{~dB}$ & $-2.2 \mathrm{~dB}$ & $5.2 \mathrm{~dB}$ & $1.2 \mathrm{~dB}$ \\
\hline LO Power & $-2.5 \mathrm{dBm}$ & $7.5 \mathrm{dBm}$ & $-3 \mathrm{dBm}$ & $0.1 \mathrm{dBm}$ \\
\hline $\mathrm{I} / \mathrm{OP} \mathrm{P}_{1 \mathrm{~dB}}$ & $-7 \mathrm{dBm}$ & $-6.5 \mathrm{dBm}$ & $-10.5 \mathrm{dBm}$ & $-9.5 \mathrm{dBm}$ \\
\hline $\mathrm{I} / \mathrm{O} \mathrm{IP}_{3}$ & $4 \mathrm{dBm}$ & $4 \mathrm{dBm}$ & $-2 \mathrm{dBm}$ & $-0.5 \mathrm{dBm}$ \\
\hline $\begin{array}{c}\text { Sideband } \\
\text { Rejection Ratio } \\
\text { Bandwidth } \\
(>30 \mathrm{~dB})\end{array}$ & $200 \mathrm{MHz}$ & $720 \mathrm{MHz}$ & & \\
\hline $\begin{array}{l}\text { Max. Sideband } \\
\text { Rejection Ratio }\end{array}$ & $52.59 \mathrm{~dB}$ & $62.85 \mathrm{~dB}$ & & \\
\hline $\begin{array}{l}\text { RF Operation } \\
\text { Frequency } \\
\left(\begin{array}{c}\text { errors }<0.5 \mathrm{~dB} \\
\left.<1^{\circ}\right)\end{array}\right.\end{array}$ & & & $\begin{array}{c}1.8 \sim 2.8 \\
\mathrm{GHz}\end{array}$ & $\begin{array}{c}4.8 \sim 5.6 \\
\mathrm{GHz}\end{array}$ \\
\hline $\begin{array}{l}\text { Magnitude } \\
\text { Imbalance }\end{array}$ & & & $0.204 \%$ & $0.944 \%$ \\
\hline Phase Error & & 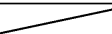 & $0.2^{\circ}$ & $0.68^{\circ}$ \\
\hline $\begin{array}{c}\text { RF Input } \\
\text { Return Loss }\end{array}$ & $-4.62 \mathrm{~dB}$ & $-4.7 \mathrm{~dB}$ & $-21.65 \mathrm{~dB}$ & $-17.31 \mathrm{~dB}$ \\
\hline $\begin{array}{c}\text { LO Input } \\
\text { Return Loss }\end{array}$ & $-12.4 \mathrm{~dB}$ & $-13.4 \mathrm{~dB}$ & $-12.45 \mathrm{~dB}$ & $-11.78 \mathrm{~dB}$ \\
\hline $\begin{array}{c}\text { IF Input Return } \\
\text { Loss }\end{array}$ & $-10.8 \mathrm{~dB}$ & $-13.3 \mathrm{~dB}$ & $-9.71 \mathrm{~dB}$ & $-10.28 \mathrm{~dB}$ \\
\hline Noise Figure & 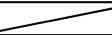 & 2 & $19 \mathrm{~dB}$ & $21 \mathrm{~dB}$ \\
\hline Supply Voltage & & & & \\
\hline $\begin{array}{c}\text { Power } \\
\text { Dissipation }\end{array}$ & $38 \mathrm{~mW}$ & $36 \mathrm{~mW}$ & $37.9 \mathrm{~mW}$ & $33.3 \mathrm{~mW}$ \\
\hline Chip Size & $1 \mathrm{mn}$ & $\mathrm{mm}$ & $1 \mathrm{~mm}$ & $1 \mathrm{~mm}$ \\
\hline
\end{tabular}

\section{CONCLUSION}

This paper has proposed a dual-band adjustable I/Q generator with quadrature phase property and constant input resistance at all frequencies. Dual band is achieved because the magnitude balance of the I/Q generator occurs at two designed frequencies. The 2.4/5.2-GHz I/Q down-converter and 2.4/5.7-GHz SSB up-converter implemented using 0.35- $\mu \mathrm{m} \mathrm{SiGe} \mathrm{BiCMOS} \mathrm{has}$ presented excellent quadrature performance of the dual-band adjustable I/Q generator. In comparison with the polyphase filter, there is no resistive loss in this generator and a multiband 
quadrature mechanism is feasible by increasing the order of the $L-C$ networks.

\section{ACKNOWLEDGMENT}

The authors would like to thank the National Chip Implementation Center (CIC), Hsinchu, Taiwan, R.O.C., for technical support.

\section{REFERENCES}

[1] K. R. Rao, J. Wilson, and M. Ismail, "A CMOS RF front-end for a multistandard WLAN receiver," IEEE Microw. Wireless Compon. Lett., vol. 15 , no. 5, pp. 321-323, May 2005.

[2] H. Hashemi and A. Hajimiri, "Concurrent multiband low-noise amplifiers-theory, design, and applications," IEEE Trans. Microw. Theory Tech., vol. 50, no. 1, pp. 288-301, Jan. 2002.

[3] L. Sheng, J. C. Jensen, and L. E. Larson, "A wide-bandwidth Si/SiGe HBT direct conversion sub-harmonic mixer/downconverter," IEEE J. Solid-State Circuits, vol. 35, no. 9, pp. 1329-1337, Sep. 2000.

[4] K. Hettak, G. A. Morin, and M. G. Stubbs, "A novel miniature multilayer MMIC CPW single side band CPW mixer for up conversion at 44.5 GHz," IEEE Microw. Wireless Compon. Lett., vol. 15, no. 9, pp. 606-608, Sep. 2005.

[5] J. C. Rundell, J.-J. Ou, T. B. Cho, G. Chien, F. Brianti, J. A. Weldon, and P. R. Gray, "A $1.9 \mathrm{GHz}$ wideband IF double conversion CMOS integrated receiver for cordless telephone applications," IEEE J. SolidState Circuits, vol. 32, no. 12, pp. 2071-1088, Dec. 1997.

[6] F. Behbahani, Y. Kishigami, J. Leete, and A. A. Abidi, "CMOS mixers and polyphase filters for large image rejection," IEEE J. Solid-State Circuits, vol. 36, no. 6, pp. 873-887, Jun. 2001.

[7] D. I. Sanderson, R. M. Svitek, and S. Raman, "A 5-6-GHz polyphase filter with tunable I/Q phase balance," IEEE Microw. Wireless Compon. Lett., vol. 14, no. 7, pp. 364-366, Jul. 2004.

[8] J. D. van der Tang, D. Kasperkovitz, and A. van Roermund, "A 9.8-11.5-GHz quadrature ring oscillator for optical receivers," IEEE J. Solid-State Circuits, vol. 37, no. 3, pp. 438-442, Mar. 2002.

[9] Z. Zhang, Z. Chen, L. Tsui, and J. Lau, "A $930 \mathrm{MHz}$ CMOS DC-offsetfree direct-conversion 4-FSK receiver," in IEEE Int. Solid-State Circuits Tech. Conf. Dig., Feb. 2001, pp. 290-291.

[10] A. A. Abidi, "Phase noise and jitter in CMOS ring oscillators," IEEE J. Solid-State Circuits, vol. 41, no. 8, pp. 1803-1816, Aug. 2006.

[11] F. Gruson, G. Gaborit, P. Abele, and H. Schumacher, "A broadband SiGe mixer for 5-GHz WLAN applications with $X$-band quadrature generation and high image-rejection," in IEEE Radio Freq. Integr. Circuits Symp., Fort Worth, TX, Jun. 2004, pp. 523-526.

[12] C.-Y. Chi and G. M. Rebeiz, "Design of Lange-couplers and singlesideband mixers using micromachining techniques," IEEE Trans. Microw. Theory Tech., vol. 45, no. 2, pp. 291-294, Feb. 1997.

[13] R. C. Frye, S. Kapur, and R. C. Melville, "A 2-GHz quadrature hybrid implemented in CMOS technology," IEEE J. Solid-State Circuits, vol. 38, no. 3, pp. 550-555, Mar. 2003.

[14] H.-C. Chen, T. Wang, S.-S. Lu, and G.-W. Huang, "A monolithic 5.9-GHz CMOS I/Q direct-down converter utilizing a quadrature coupler and transformer-coupled subharmonic mixers," IEEE Microw. Wireless Compon. Lett., vol. 16, no. 4, pp. 197-199, Apr. 2006.

[15] P. Andreani and X. Wang, "On the phase-noise and phase-error performances of multiphase LC CMOS VCOs," IEEE J. Solid-State Circuits, vol. 39, no. 11, pp. 1883-1893, Nov. 2004.
[16] P. Andreani, A. Bonfanti, L. Ramano, and C. Samori, "Analysis and design of a 1.8-GHz CMOS LC quadrature VCO," IEEE J. Solid-State Circuits, vol. 37, no. 12, pp. 1737-1747, Dec. 2002.

[17] S. L. J. Gierkink, S. Levantino, R. C. Frye, C. Samori, and V. Boccuzzi, "A low-phase-noise 5-GHz CMOS quadrature VCO using superharmonic coupling," IEEE J. Solid-State Circuits, vol. 38, no. 7, pp. 1148-1154, Jul. 2003.

[18] Y. Itoh, M. Nii, Y. Kohno, M. Mochizuki, and T. Takagi, "A 4 to 25 $\mathrm{GHz} 0.5 \mathrm{~W}$ monolithic lossy match amplifier," in IEEE MTT-S Int. Microw Symp. Dig., San Diego, CA, May 1994, pp. 257-260.

[19] B. Gilbert, "The MICROMIXER: A highly linear variant of the Gilbert mixer using a bisymmetric class-AB input stage," IEEE J. Solid-State Circuits, vol. 32, no. 9, pp. 1412-1423, Sep. 1997.

[20] S. C. Tseng, C. C. Meng, C. H. Chang, C. K. Wu, and G. W. Huang, "Monolithic broadband Gilbert micromixer with an integrated Marchand balun using standard silicon IC process," IEEE Trans. Microw. Theory Tech., vol. 54, no. 12, pp. 4362-4371, Dec. 2006.

[21] C. C. Meng, S. K. Hsu, A. S. Peng, S. Y. Wen, and G. W. Huang, "A fully integrated $5.2 \mathrm{GHz}$ GaInP/GaAs HBT upconversion micromixer with output $L C$ current combiner and oscillator," in IEEE MTT-S Int. Microw Symp. Dig., Philadelphia, PA, Jun. 2003, pp. A205-A208.

[22] J. Janssens and M. Steyaert, CMOS Cellular Receiver Front-Ends. Boston, MA: Kluwer, 2002, ch. 3, sec. 3.5.5, pp. 43-44.

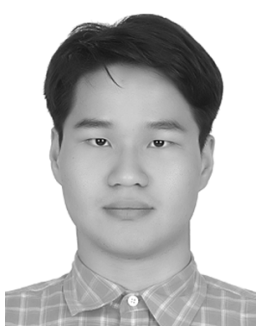

Sheng-Che Tseng (S'05) received the B.S. degree in communication engineering from National Chiao Tung University, Hsinchu, Taiwan, R.O.C., in 2003, and is currently working toward the Ph.D. degree in communication engineering at National Chiao Tung University.

His current research focuses on RFICs and highfrequency circuitry.

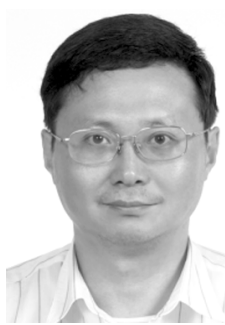

Chinchun Meng (M'02) received the B.S. degree in electrical engineering from National Taiwan University, Taipei, Taiwan, R.O.C., in 1985, and the Ph.D. degree in electrical engineering from the University of California at Los Angeles (UCLA), in 1992.

$\mathrm{He}$ is currently a Full Professor with the Department of Communication Engineering, National Chiao Tung University, Hsinchu, Taiwan, R.O.C. His current research interests are in the areas of RFICs, high-frequency circuits, and high-speed devices.

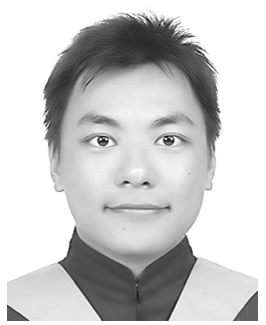

Yueh-Ting Lee was born in Taipei, Taiwan, R.O.C., in 1982. He received the B.S. degree in electrical engineering from National Cheng Kung University, Taiwan, R.O.C., in 2005, and the M.S. degree in communication engineering from National Chiao Tung University. Hsinchu, Taiwan, R.O.C., in 2007.

He was involved with SiGe HBT mixers, low-noise amplifiers, and pseudomorphic HEMT (pHEMT) $60-\mathrm{GHz}$ amplifiers during his masters research. 